\title{
Apport de l'observation satellitaire à l'analyse et à la prévision de la qualité de l'air
}

\section{Résumé}

Cet article présente ce qu'apportent les observations satellitaires pour les études de la composition atmosphérique, notamment dans un cadre d'analyse et prévision de la qualité de l'air. Les différentes mesures aujourd'hui disponibles sont présentées avec leurs principales forces et limitations. ̀̀ partir d'exemples d'études récentes, l'utilisation et le gain obtenu grâce à ces observations sont discutés. Cela inclut l'analyse d'événements de transports de panaches de polluants, le raffinement des bases de données utilisées dans les modèles, la modélisation des observables satellitaires et l'assimilation de données. Le récent service Copernicus atmosphère est détaillé dans ce contexte et les perspectives futures d'utilisation de ces observations sont finalement expliquées.

\section{Abstract}

\section{Contribution of satellite observation to air quality analysis and forecast}

This paper presents what can be achieved with the use of satellite observations in the framework of atmospheric composition studies, including for air quality analysis and forecast. The different measurements currently available are presented with their main strengths and limitations. On the basis of examples from recent studies, the use and the gain obtained by these observations are discussed. This includes analysis of pollutant transport events, refinement of the databases used in the models, modeling of satellite observations and data assimilation. The recent Copernicus service is detailed in this context and the prospects for using these observations are finally explained.

\author{
Laurent Menut ${ }^{1}$, Jean-Luc Attié2,7, Matthias Beekmann ${ }^{3}$, \\ Bertrand Bessagnet $^{4}$, Cathy Clerbaux ${ }^{5}$, Juan Cuesta ${ }^{3}$, Gaelle Dufour ${ }^{3}$, \\ Gilles Forêt ${ }^{3}$, Laaziz El Amraoui ${ }^{2}$, Marc Mallet ${ }^{2}$, Frédérik Meleux ${ }^{4}$, \\ Philippe Ricaud $^{2}$, Didier Tanré ${ }^{-6}$, Solène Turquety ${ }^{1}$ \\ 1 Laboratoire de météorologie dynamique, Institut Pierre-Simon Laplace, \\ École polytechnique / Université Pierre-et-Marie-Curie / CNRS, Palaiseau \\ 2 Centre national de la recherche météorologique, Météo-France \\ CNRS, Toulouse \\ 3 Laboratoire interuniversitaire des systèmes atmosphériques, \\ Université Paris-Est-Créteil/Université Paris-Diderot, Créteil \\ 4 Institut national de l'environnement industriel et des risques, \\ Parc technologique Alata, Verneuil-en-Halatte \\ 5 Laboratoire atmosphères et observations spatiales, \\ Institut Pierre-Simon Laplace, Université Pierre-et-Marie-Curie / CNRS, Paris \\ 6 Laboratoire d'optique atmosphérique, Université de Lille / CNRS, Lille \\ 7 Laboratoire d'aérologie, Université de Toulouse / CNRS, Toulouse
}

menut@lmd.polytechnique.fr

a recherche en physico-chimie de

l'atmosphère s'appuie à la fois sur des observations et de la modélisation. Il est nécessaire de comprendre le cycle de vie des espèces gazeuses et des aérosols pour mieux quantifier les impacts de ces espèces chimiques sur la santé humaine, les écosystèmes et le climat. En ce qui concerne la qualité de l'air, les concentrations sont considérées comme excessives lorsqu'elles dépassent des seuils établis à partir de concentrations de fond caractéristiques d'une atmosphère propre : on parle alors de polluants atmosphériques. Ces polluants peuvent avoir des origines et des modes d'émissions très divers avec des sources aussi bien naturelles qu'anthropiques. Lorsqu'ils dépassent des seuils réglementaires, on parle alors de dégradation de la qualité de l'air. Celle-ci est surveillée depuis de nombreuses années, à la fois par des réseaux délivrant des mesures de surface et au moyen de campagnes de mesures sur des régions et des périodes particulières. Les réseaux de surveillance disponibles en Europe apportent la connaissance toutes les heures des concentrations en surface de l'ozone $\mathrm{O}_{3}$, du monoxyde de carbone $\mathrm{CO}$, des oxydes d'azote $\mathrm{NO}_{\mathrm{x}}$ et du dioxyde de soufre $\mathrm{SO}_{2}$ pour les gaz et des $\mathrm{PM}_{2.5}$ et $\mathrm{PM}_{10}$ pour les aérosols (particules dont le diamètre est inférieur à respectivement 2,5 et $10 \mu \mathrm{m}$ ). Les recherches menées ces dernières décennies ont montré toute la complexité du système : il existe un grand nombre d'espèces chimiques différentes dont les sources et les puits sont très variés. Afin de progresser sur la connaissance de leurs cycles de vie, et pour obtenir des informations complémentaires aux observations de surface, les mesures satellitaires sont de plus en plus analysées et utilisées pour caractériser des situations de pollution atmosphérique.

Les mesures satellitaires de composition chimique de la troposphère ont tout d'abord été utilisées à l'échelle globale. Peu résolues spatialement et temporellement, plutôt qualitatives, elles ont permis de décrire le transport 
de panaches de polluants à longue distance, pour des espèces ayant une durée de vie atmosphérique de quelques mois. L'amélioration des mesures et les progrès réalisés sur l'algorithmie d'analyse permettent aujourd'hui d'apporter de l'information précise aux échelles régionales, voire métropolitaines. En renseignant des zones peu ou pas couvertes par les mesures in situ, les observations satellitaires peuvent être utilisées à différents niveaux : elles peuvent servir à contraindre les inventaires d'émissions de polluants, permettre une analyse fine de résultats de simulation sur de vastes régions et sur toute la colonne atmosphérique, ou encore être assimilées par les modèles pour améliorer la performance de ceux-ci.

\section{Les différentes}

\section{mesures satellitaires actuelles de la pollution atmosphérique}

De nombreux capteurs existent aujourd'hui, chacun avec des caractéristiques techniques permettant de discriminer une ou plusieurs espèces chimiques atmosphériques, avec des fréquences de mesures et des couvertures spatiales différentes. Une synthèse de ces missions spatiales en cours est présentée dans le tableau 1. De ces informations, on peut déduire dans les grandes lignes les avantages et inconvénients des capteurs existants pour les études de pollution atmosphérique régionale.

La plupart des polluants que 1'on cherche à estimer sont aujourd'hui mesurés par au moins un capteur. Cependant, si la quantification des espèces gazeuses a atteint un bon niveau de précision, ce n'est pas le cas pour les aérosols, souvent uniquement représentés sous la forme de leur effet sur l'épaisseur optique (AOD, Aerosol Optical Depth) sans discriminer les espèces présentes ni la distribution en taille des particules (par exemple $\mathrm{PM}_{2.5}$ et $\left.\mathrm{PM}_{10}\right)$. Par contre, la multiplicité de satellites différents peut permettre de raffiner l'évaluation d'une concentration par recoupements entre les mesures.

L'indéniable intérêt de l'observation par satellite est de pouvoir réaliser des mesures sur l'ensemble du globe, indépendamment de l'installation d'un réseau de mesures au sol. Un désavantage est de ne souvent disposer que de mesures intégrées sur la verticale, ce qui en limite fortement l'intérêt pour l'étude de la qualité de l'air qui nécessite la connaissance des concentrations de polluants à proximité de la surface. Par ailleurs, les mesures satellitaires sont potentiellement gênées par la couverture nuageuse ou par des albédos de surface importants (notamment pour l'inversion des aérosols). Enfin, si l'on cherche à caractériser finement un événement de pollution sur une zone urbanisée particulière, leur résolution spatiale ( $\sim 10 \mathrm{~km}$ au mieux) et temporelle (une à deux mesures par jour) reste généralement trop faible.

Pour bien comprendre un événement de pollution, sur une durée de quelques jours, ces données vont pouvoir être exploitées en utilisant différentes méthodes. On peut tout d'abord privilégier l'analyse d'événements en s'aidant d'espèces chimiques à longue durée de vie et avec une signature spectrale nette. On peut aussi combiner différents capteurs afin de réduire l'incertitude liée à chacun. Quelle que soit l'approche, la modélisation permet d'apporter une information supplémentaire et d'affiner la compréhension de l'événement étudié. L'étude des événements de pollution peut aussi consister à réaliser des études de tendance : le manque de couverture spatiale et temporelle est alors compensé par l'accumulation de données et leur exploitation statistique.

\section{Analyse d'événements de transports de panaches}

Les panaches de polluants résultent de fortes émissions de gaz et d'aérosols qui sont concentrées spatialement et/ ou temporellement : les émissions peuvent être d'origine anthropique (comme les grandes mégapoles) ou naturelle (volcans, feux de végétation, soulèvement de poussières minérales). Le suivi par satellite de ces panaches permet, en première approximation, de faire la part entre polluants anthropiques et naturels. Cette distinction est indispensable, car la pollution sur laquelle on peut agir est principalement d'origine anthropique. Cette différenciation entre pollution anthropique et naturelle est également pertinente dans le cadre de la directive européenne de la qualité de l'air 2008/50/CE. En effet, celle-ci permet aux États membres de retrancher les contributions d'origine naturelle des concentrations totales de polluants mesurées, ce qui peut avoir pour conséquence de diminuer le nombre de dépassement des seuils réglementaires à déclarer aux autorités européennes.

\section{Mieux comprendre des événements de dispersion régionale}

Les feux de végétation sont un bon exemple d'événements importants de pollution atmosphérique pour lesquels il est difficile de réaliser des mesures in situ ou aéroportées. Le satellite apporte alors une information précieuse.

Récurrents chaque été, les feux en Californie ont par exemple été observés par satellite et simulés pour l'été 2008. La figure 1 montre l'épaisseur optique (AOD) estimée à partir des observations satellitaires (capteur Modis) dans le visible $(550 \mathrm{~nm})$ pour la date du 13 juillet 2008, ainsi que celle calculée à l'aide du modèle régional RegCM. Le satellite

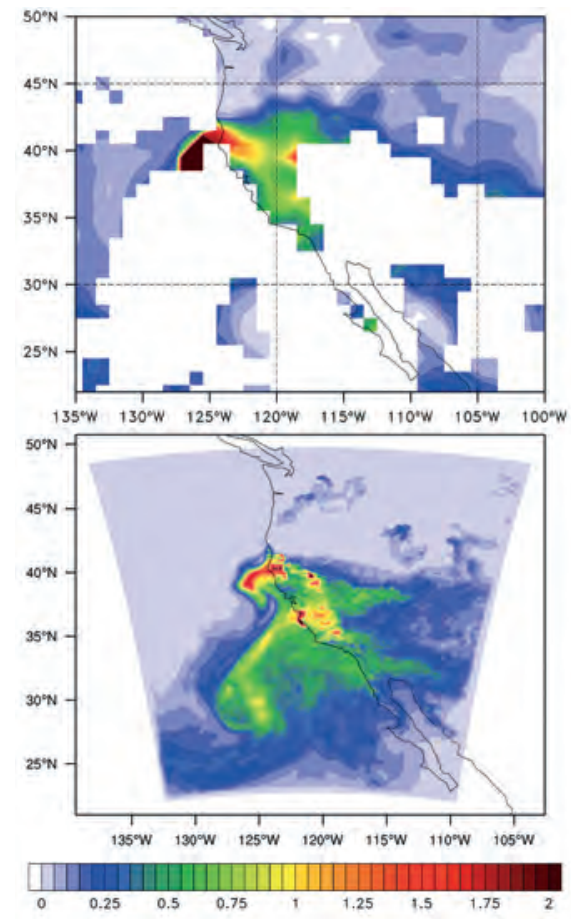

Figure 1. Épaisseur optique en aérosols à $550 \mathrm{~nm}$ observée (Modis-Aqua, en haut) et simulée (modèle RegCM, en bas), le 13 juillet 2008 sur et au large de la région californienne. 
Tableau 1. Principales caractéristiques des missions satellitaires pour la mesure des polluants (TIR, thermal IR, infrarouge thermique; NIR, near IR, proche infrarouge ; VIS, visible ; UV : ultraviolet).

\begin{tabular}{|c|c|c|c|c|}
\hline Satellite & $\begin{array}{l}\text { Nom } \\
\text { du sondeur } \\
\text { Longueurs } \\
\text { d'onde }\end{array}$ & $\begin{array}{l}\text { Spécifications } \\
\text { instrumentales }\end{array}$ & Polluant mesuré & Commentaires \\
\hline & $\begin{array}{l}\text { Mopitt } \\
\text { TIR + NIR }\end{array}$ & $\begin{array}{l}\text { Spectromètre à corrélation } \\
\text { Pixel : } 22 \text { × } 22 \mathrm{~km}^{2} \\
\text { Revisite } 3 \text { jours }\end{array}$ & $\mathrm{CO}$ & $\begin{array}{l}\text { Données homogènes depuis } 2000 \\
1-2 \text { informations verticales } \\
\text { indépendantes }\end{array}$ \\
\hline 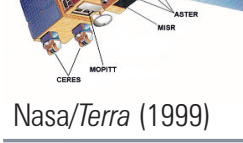 & $\begin{array}{l}\text { Modis } \\
\text { Visible }\end{array}$ & $\begin{array}{l}\text { Spectromètre imageur } \\
\text { Pixel : 250-1 } 000 \mathrm{~m} \\
\text { Fauchée : } 2330 \mathrm{~km}\end{array}$ & $\begin{array}{l}\text { Aérosol : type, AOD, } \\
\text { taille, propriétés } \\
\text { optiques }\end{array}$ & $\begin{array}{l}\text { Permet de différencier } \\
\text { nuages/particules }\end{array}$ \\
\hline & $\begin{array}{l}\text { Airs } \\
\text { TIR }\end{array}$ & $\begin{array}{l}\text { Spectromètre à réseau } \\
\text { Pixel : } 13,5 \times 13,5 \mathrm{~km}^{2} \\
\text { Fauchée : } 1650 \mathrm{~km}\end{array}$ & $\mathrm{CO}, \mathrm{O}_{3}, \mathrm{NH}_{3}$ & $\begin{array}{l}\text { Couverture globale } \\
\text { Mesure jour + nuit } \\
\text { résolution verticale limitée }\end{array}$ \\
\hline Nasa/Aqua (2002) & $\begin{array}{l}\text { Modis } \\
\text { Visible }\end{array}$ & $\begin{array}{l}\text { Spectromètre imageur } \\
\text { 250-1000 m } \\
\text { Fauchée : } 2330 \text { km }\end{array}$ & $\begin{array}{l}\text { Aérosol : type, AOD, } \\
\text { taille, propriétés } \\
\text { optiques }\end{array}$ & $\begin{array}{l}\text { Permet de différencier } \\
\text { nuages/particules }\end{array}$ \\
\hline & $\begin{array}{l}\text { OMl } \\
\text { UV-vis }\end{array}$ & $\begin{array}{l}\text { Spectromètre UV-vis } \\
13 \mathrm{~km} \times 12 \text { / } 24 \text { / } 48 \mathrm{~km} \\
\text { Fauchée : } 2600 \mathrm{~km}\end{array}$ & $\mathrm{NO}_{2}, \mathrm{O}_{3}, \mathrm{SO}_{2}, \mathrm{AOD}$ & $\begin{array}{l}\text { Couverture globale } \\
\text { Résolution verticale limitée }\end{array}$ \\
\hline Nasa/Aura (2004) & $\begin{array}{l}\text { TES } \\
\text { TIR }\end{array}$ & $\begin{array}{l}\text { Spectromètre } \\
\text { à transformée de Fourier } \\
\text { Pixel : } 5,3 \times 8,3 \mathrm{~km}\end{array}$ & $\mathrm{CO}, \mathrm{O}_{3}, \mathrm{NH}_{3}, \mathrm{SO}_{2}$ & $\begin{array}{l}\text { Couverture horizontale limitée } \\
\text { Mesure jour et nuit } \\
\text { Bonne résolution spectrale }\end{array}$ \\
\hline
\end{tabular}

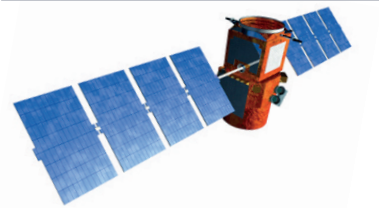

Caliop + IRR
(A-train)

Lidar (polarisation

orthogonale) + imageur

Profils verticaux

aérosols et nuages

Excellente résolution verticale mais mauvaise couverture horizontale

Cnes/Nasa Calipso (2006)

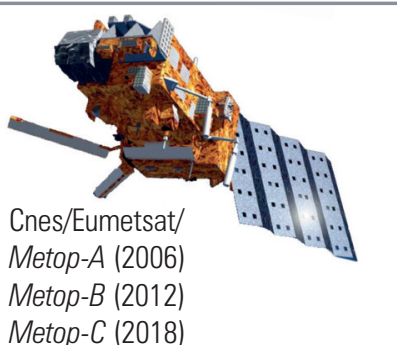

\begin{tabular}{llll}
$\begin{array}{l}\text { Gome-2 } \\
\text { UV-vis }\end{array}$ & $\begin{array}{l}\text { Spectromètre UV-vis } \\
\text { Pixel : } 40 \times 40 \mathrm{~km}^{2} \\
\text { Fauchée }: 1920 \mathrm{~km}\end{array}$ & $\mathrm{NO}_{2}, \mathrm{O}_{3}, \mathrm{SO}_{2}, \mathrm{CH}_{2} \mathrm{O}$ & $\begin{array}{l}\text { Bonne couverture horizontale } \\
\text { mais large pixel }\end{array}$ \\
\hline lasi & $\begin{array}{l}\text { Spectromètre TF } \\
\text { TIR }\end{array}$ & $\mathrm{CO}_{1} \mathrm{O}_{3}, \mathrm{NH}_{3}, \mathrm{SO}_{2}$, & $\begin{array}{l}\text { Très bonne couverture horizontale, } \\
\text { mesure jour et nuit, } \\
\text { résolution verticale limitée }\end{array}$
\end{tabular}

$\begin{array}{ll}\text { OMPS } & \text { Spectromètre UV } \\ \text { UV } & \text { Pixel : } 50 \mathrm{~km} \\ & \text { Fauchée : } 2800 \mathrm{~km}\end{array}$

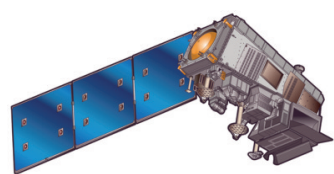

Suomi NPP (2011)
Cris

TIR

Fauchée : 2800 km

Spectromètre à réseau $\quad \mathrm{O}_{3}, \mathrm{NH}_{3}$, (CO à venir)

Pixel : $13,5 \times 13,5 \mathrm{~km}^{2}$

Fauchée : $2200 \mathrm{~km}$

$\begin{array}{ll}\text { VIIRS } & \text { Spectromètre imageur } \\ \text { Visible } & \text { Pixel : } 12 \mathrm{~km} \\ & \text { Fauchée : } 3040 \mathrm{~km}\end{array}$

Aérosol : type, AOD, taille, propriétés optiques

\section{$\mathrm{O}_{3}$ \\ Continuité missions, \\ mesure de la colonne totale}

Bonne couverture horizontale, mesure jour et nuit, résolution verticale limitée

Permet de différencier nuages/particules

$\begin{array}{ll}\text { Polder } & \text { Radiomètre imageur } \\ & \text { à grand champ } \\ & \text { Pixel : } 6 \mathrm{~km} \\ & \text { Fauchée }: 1600 \mathrm{~km}\end{array}$

Fauchée : $1600 \mathrm{~km}$
Aérosol : type, AOD, taille, propriétés optiques
Mesure les caractéristiques directionnelles et polarisées de la lumière réfléchie par l'ensemble Terre-atmosphère 
permet de confirmer que le modèle reproduit correctement la structure des panaches de feux de végétation. Mais la figure montre aussi les limitations du satellite dans ce cas : les stratocumulus marins fréquents au large de la Californie ne permettent pas d'avoir toute l'information sur la couche d'aérosols en la masquant partiellement. Il est alors difficile de conclure sur la qualité du modèle sur ces régions spécifiques. Une piste d'amélioration est certainement le développement de nouveaux produits «aérosols» en présence de nuages, comme celui récemment développé pour l'instrument Polder (Waquet et al., 2013).

Un autre exemple est le suivi du monoxyde de carbone (CO) qui joue un rôle central dans la chimie troposphérique, car il est détruit par le radical $\mathrm{OH}$ et régule dès lors la capacité oxydante de 1'atmosphère. Dans la troposphère, il est émis principalement par les activités anthropiques (transport, chauffage, industrie) et par les feux de végétation (naturels ou provoqués par l'Homme). La surveillance du CO comme polluant atmosphérique local/régional n'est pas une priorité en Europe, car, contrairement à l'Asie, par exemple, les normes de qualité de l'air ne sont jamais dépassées pour ce gaz. Par contre, les satellites apportent une information pertinente pour le suivi du transport des masses d'air pollué.

Les concentrations de $\mathrm{CO}$ dans l'atmosphère sont très variables : le niveau de fond loin des sources, dû à la photodissociation du méthane, est d'environ $50 \mathrm{ppb}^{1}$ à la surface, tandis que des concentrations dépassant 1000 ppb sont observées dans les zones très polluées (figure $2 \mathrm{a}$ ). Cette variabilité spatiale et saisonnière en fait un composé parfait pour le suivi par satellite. L'instrument Mopitt (Deeter et al., 2015) offre des cartes journalières depuis 2000 et les deux instruments Iasi complètent la série depuis 2006, avec deux à quatre mesures par jour en tout point du globe (George et al., 2015). Des concentrations particulièrement élevées ont ainsi été mesurées par Iasi lors des grands feux de l'automne 2015 qui ont ravagé l'Indonésie suite à la sécheresse intense liée à un épisode $\mathrm{El}$ Niño (figure $2 b$ ).

Les éruptions volcaniques sont un autre exemple d'émissions naturelles de polluants pour lesquelles le satellite

1. Partie par milliard, soit une molécule sur un milliard. apporte une information importante. Les observations satellitaires permettent de comprendre l'étendue de l'événement de transport de pollution associé et de mesurer les variations de la composition atmosphérique. De récentes études ont combiné différents capteurs pour obtenir une vue d'ensemble de l'épisode : imagerie, AOD par Modis et Seviri, profil lidar Caliop, dioxyde de soufre et cendres par Iasi. Cela a permis de reconstruire les flux d'émissions et leur variabilité, permettant une

quantification de leur impact sur la qualité de l'air régional (voir par exemple Chazette et al., 2012 ; Boichu et al., 2015).

\section{Mieux comprendre les structures verticales}

L'origine de panaches peut être difficile à interpréter à partir des seules observations en surface et le satellite peut s'avérer très utile. Par exemple, en
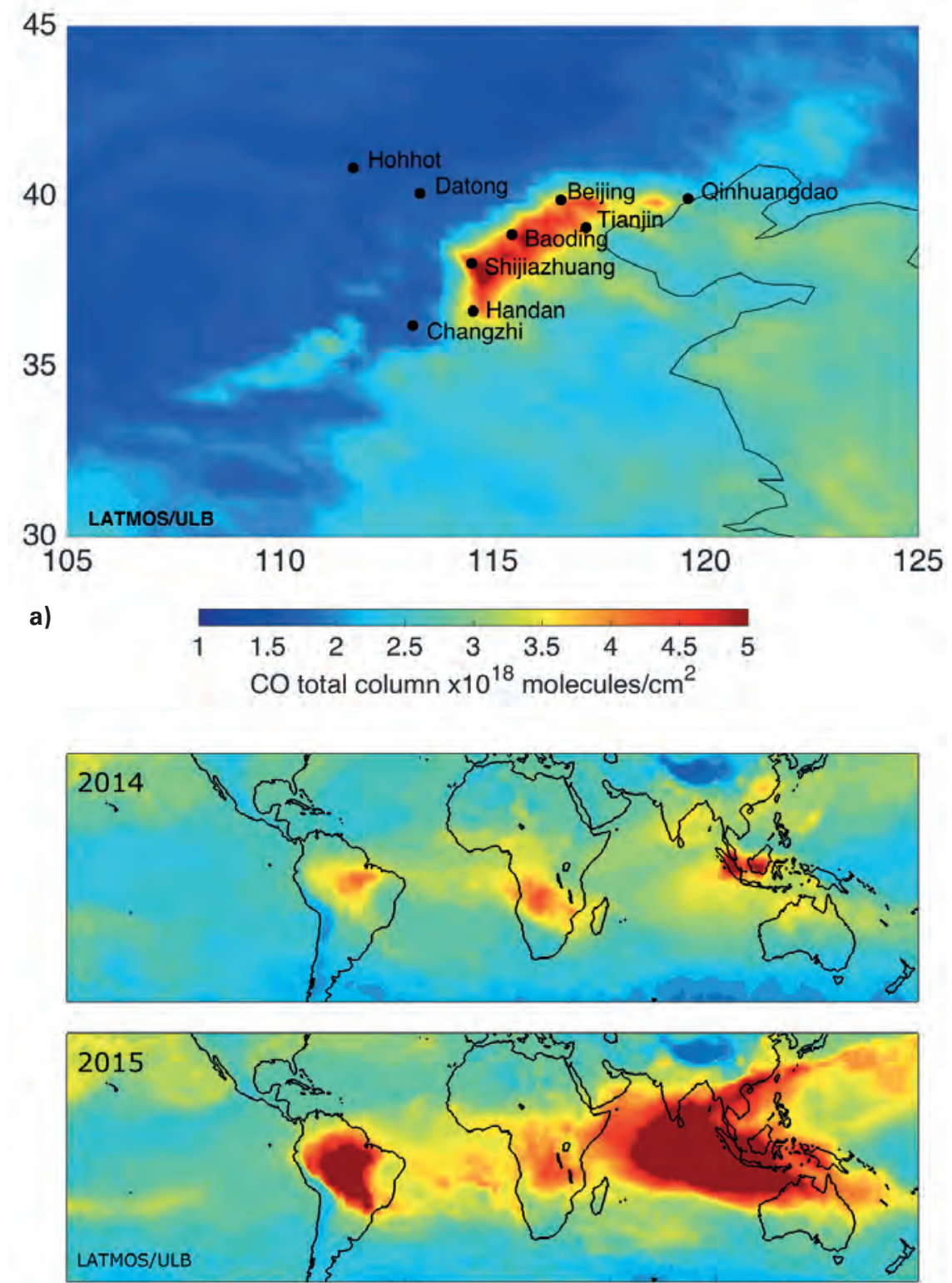

b)

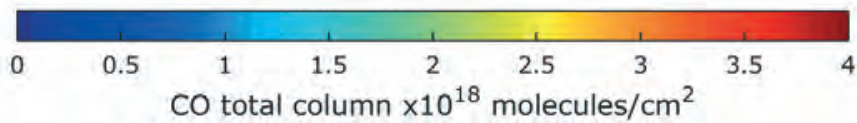

Figure 2. (a) Colonne de $\mathrm{CO}$ (molécules $\mathrm{cm}^{-2}$ ) observée par l'instrument lasi à bord du satellite Metop durant un épisode de pollution (Chine, décembre 2015). La carte a été obtenue suivant la méthodologie décrite dans Boynard et al. (2014). (b) Colonne de CO observée par lasi pour deux périodes de 6 jours en novembre 2014 et 2015. Les cartes mettent en évidence le transport du CO émis par les feux de végétation qui se produisent de manière récurrente en automne dans I'hémisphère Sud. En 2015, la sécheresse intense liée à El Niño a conduit à des feux d'une intensité exceptionnelle. Crédit : Maya George (Latmos). 
mars 2007, un épisode de pollution de l'Ukraine jusqu'en Europe de l'Ouest avait tout d'abord été attribué à un soulèvement d'aérosols minéraux au Sahara. En Europe centrale, des concentrations de surface de $\mathrm{PM}_{10}$ supérieures à $1000 \mu \mathrm{g} \mathrm{m}^{-3}$ ont été mesurées et le nord de la France a été affecté par des concentrations supérieures à $150 \mu \mathrm{g} \mathrm{m}^{-3}$. Grâce aux données de Caliop et à sa capacité à fournir des profils verticaux d'aérosols, l'origine ukrainienne, liée à des sols chernozémiques très érodables, a pu être établie avec certitude, contredisant une lecture trop hâtive d'un simple calcul de rétrotrajectoires (Bessagnet et al., 2008).

La figure 3 montre un autre exemple de transport d'aérosols minéraux, cette fois observé en Asie en mars 2008. Caliop a permis de détecter une tempête de poussières pendant cette période. Ces poussières ont été soulevées dans les déserts mongoliens et transportées à l'échelle du continent, perturbant les activités humaines dans toute l'Asie de l'Est. Les traces journalières étant assez distantes (24 degrés en moyenne), il est difficile d'établir des cartes de suivis. Par contre, la résolution verticale (quelques dizaines de mètres) permet de restituer finement la variabilité verticale des panaches. De manière complémentaire, les sondeurs hyperspectraux passifs, comme Iasi (Clerbaux et al., 2009), ont montré des capacités pour restituer la distribution verticale des aérosols dits grossiers (typiquement pour un diamètre supérieur à $1 \mu \mathrm{m})$ comme les poussières minérales (Cuesta et al., 2015). La figure 3 montre que les deux instruments restituent les mêmes structures verticales en termes d'extinction pour des longueurs d'onde différentes. En complément, les observations permettent de voir que les sources d'aérosols minéraux sont probablement bien placées dans le modèle, mais que le panache simulé en lui-même est sous-estimé en intensité ou déplacé par rapport à la trace du satellite étudiée.

\section{Combiner les capteurs pour affiner l'analyse}

Une comparaison du contenu en aérosols (exprimé en épaisseur optique à $500 \mathrm{~nm}$ ) permet d'illustrer la complémentarité et les limites des observations effectuées dans les différents domaines spectraux (UV, visible, infrarouge thermique) ou par les différents types de capteurs, en polarisation ou pas, actif ou passif. Un a)

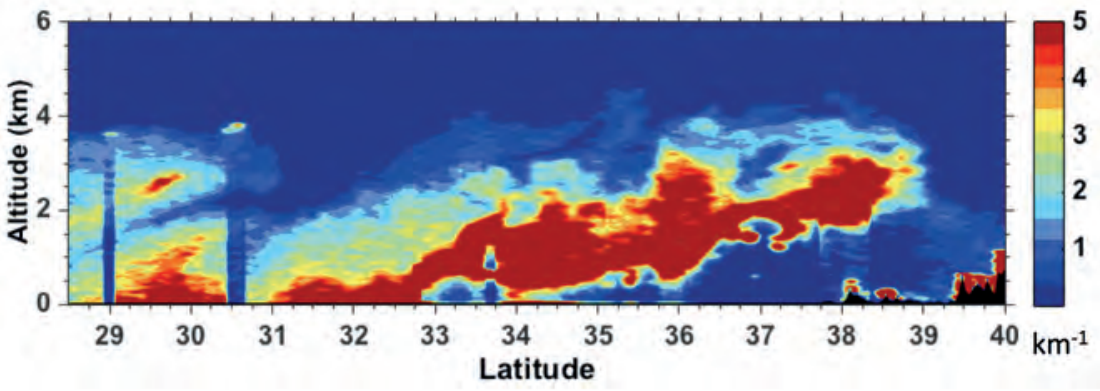

b)

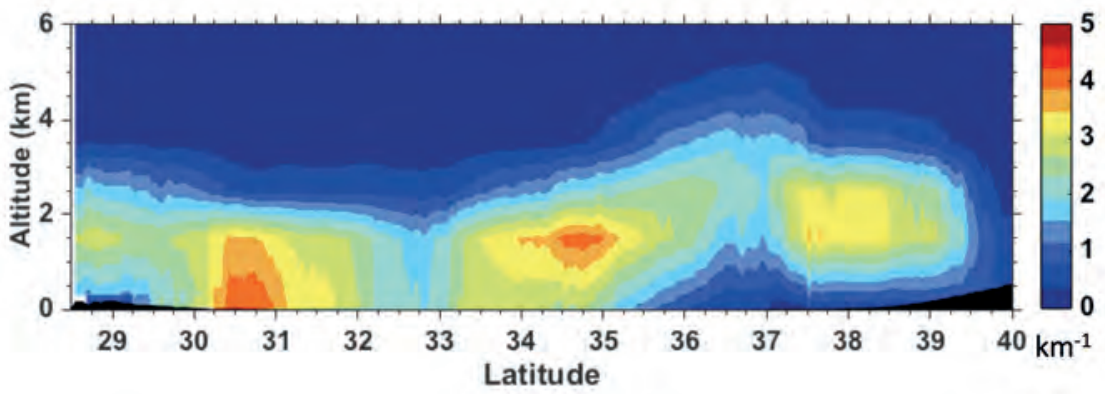

c)

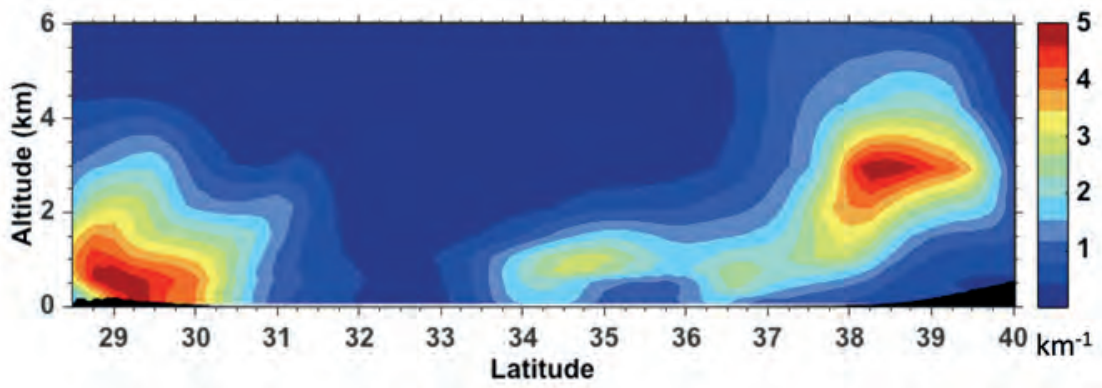

Figure 3. Analyse d'une tempête d'aérosols minéraux en Asie le 2 mars 2008. Les figures représentent des coupes verticales selon un axe sud-nord (longitude $123^{\circ} \mathrm{E}$ ) du coefficient d'extinction des aérosols (en $\mathrm{km}^{-1}$ ). (a) Inversion obtenue à partir des mesures du lidar spatial Caliop à $532 \mathrm{~nm}$ vers $17 \mathrm{~h} 30$ UTC, tenant compte de la diffusion multiple. (b) Restitution lasi à $10 \mu \mathrm{m}$ vers 12 h 30 UTC obtenue à partir de la méthode Aeroiasi. (c) Modélisation Chimere à $550 \mathrm{~nm}$ pour $18 \mathrm{~h}$ UTC.

même événement de poussières vu par les différents instruments de l'A-Train (OMI, Modis, Airs, Calipso et Parasol) au-dessus de la Méditerranée en février 2007 est reporté en figure 4. La comparaison (figure 4b) met en évidence la sensibilité de l'épaisseur optique retrouvée dans l'infrarouge par l'instrument Airs à l'altitude du panache (altitude déterminée à partir du capteur lui-même, à partir du lidar ou estimée depuis la climatologie du modèle Gocart). Comme les propriétés optiques des aérosols sont différentes dans l'infrarouge et dans le visible, les épaisseurs optiques ne sont pas directement comparables et nous avons ici utilisé un rapport 4 pour convertir l'épaisseur optique de Airs en une épaisseur optique à $500 \mathrm{~nm}$. De même, les mesures réalisées dans l'ultraviolet par l'instrument OMI nécessitent pour être interprétées en épaisseur optique de connaître l'altitude du panache du fait de la contribution importante de la diffusion Rayleigh dans cette partie du spectre et à l'effet de masque correspondant. En ce qui concerne les mesures de Caliop, la valeur du rapport lidar $^{2}$ est primordiale et, dans ce cas, la contribution des diffusions multiples (non corrigée ici) peut aussi affecter l'inversion. En ce qui concerne Modis et Parasol, les techniques d'observation, modulo la polarisation, sont similaires et les valeurs retrouvées proches; on peut simplement souligner que, Parasol disposant de plus d'observations en polarisation pour ajuster le modèle d'aérosol, les valeurs retrouvées sont $a$ priori plus proches de la réalité. Les propriétés structurelles (répartition verticale) ou microphysiques (taille,

2. Le rapport lidar désigne le rapport entre rétrodiffusion et diffusion de l'aérosol. Il est nécessaire pour résoudre l'équation du signal lidar. 
indice de réfraction) des aérosols impactant les mesures spatiales différemment suivant le domaine de longueur d'onde, il faut utiliser un modèle d'aérosols cohérent sur l'ensemble du spectre et optimiser les contraintes à exercer sur les propriétés optiques utilisées dans les différents algorithmes pour pleinement valoriser la synergie instrumentale.

\section{Analyses systématiques pour des régions particulières}

En dehors des événements de transport, plusieurs études ont utilisé les comparaisons entre observations satellitaires et simulations comme base pour analyser la pollution dans des régions soumises à de nombreuses influences. En Méditerranée, par exemple, Safieddine et al. (2014) et Doche et al. (2014) ont utilisé six ans d'observations Iasi pour analyser la variabilité de l'ozone troposphérique. Ils ont exploité les observations d'un été spécifique avec des simulations régionales pour comprendre les sources influençant les concentrations d'ozone. Ces études ont mis en évidence l'influence des sources régionales anthropiques en dessous de $4 \mathrm{~km}$ d'altitude, mais aussi l'impact d'une subsidence depuis la haute troposphère, surtout pour les parties centrale et est du bassin méditerranéen. En revanche, les concentrations en altitude sont principalement influencées par le transport provenant d'autres régions.
Toujours d'un point de vue des tendances sur de longues périodes et en Méditerranée, une analyse similaire pour les aérosols a montré une contribution majeure des poussières désertiques aux épaisseurs optiques simulées. Les résultats obtenus en termes de contributions relatives des différentes sources sont similaires pour les épaisseurs optiques et les $\mathrm{PM}_{10}$ de surface, mais différentes pour les $\mathrm{PM}_{2.5}$ qui sont quant à elles influencées majoritairement par les sources anthropiques (Rea et al., 2015).

\section{Élaborer et mettre à jour les bases de données pour les modèles}

Pour réaliser des prévisions de la qualité de l'air, les modèles de chimie-transport sont forcés par des champs météorologiques et des bases de données décrivant de nombreux paramètres surfaciques. Ces paramètres représentent, par exemple, l'occupation des sols et des surfaces (pour le calcul des émissions de minéraux, de feux), le type et l'abondance de la végétation (pour les émissions biogéniques) et l'urbanisation (pour les émissions anthropiques).

Les plus anciens modèles utilisaient des bases de données peu résolues spatialement et statiques temporellement. Les valeurs utilisées représentaient des valeurs moyennes, permettant de simuler au mieux un ensemble de situations observées. Les résultats des modèles étaient alors relativement lisses, les bases de données cherchant surtout à permettre de simuler au mieux les événements les plus fréquents, en limitant les biais dans les résultats.

\section{Améliorer les bases de données existantes}

Les données satellitaires permettent d'améliorer les bases de données existantes. Ces données donnent une information cohérente sur l'ensemble du globe et non plus limitée à un lieu. Par exemple, les calculs des émissions biogéniques ont énormément évolué en utilisant des bases de données de végétation aujourd'hui kilométriques et qui permettent de distinguer de nombreuses essences d'arbres, un paramètre particulièrement important pour le calcul des flux d'émission. Les mesures étant fréquentes, les bases de données permettent aussi de prendre en compte de manière réaliste la variabilité de l'indice foliaire (LAI, Leaf Area Index), via des bases de données de résolution mensuelle (Guenther et al., 2012). Pour les émissions anthropiques, les forcages utilisés peuvent aussi être améliorés. Aujourd'hui, la répartition spatiale des sources est plus réaliste car l'imagerie visible permet de dresser des cartes d'utilisation des sols et de localiser précisément villes, autoroutes et industries. Cependant, les valeurs des flux ne bénéficient pas encore de ces informations additionnelles. a)

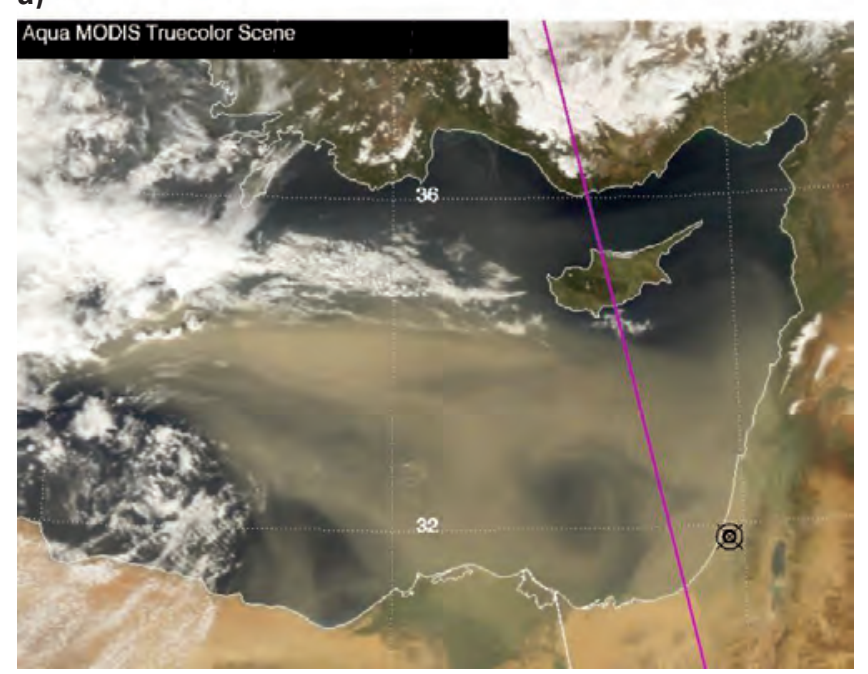

b)

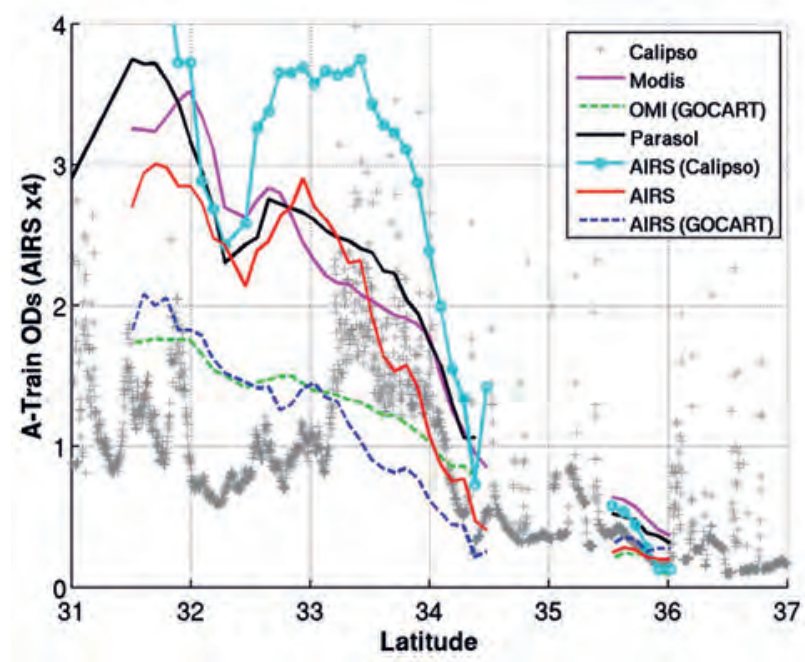

Figure 4. (a) Image en vraie couleur réalisée à partir des mesures Modis dans le visible d’un événement de poussières observé sur la partie est du bassin méditerranéen le 24 février 2007. La trace de Calipso est reportée en magenta. (b) Épaisseur optique à $500 \mathrm{~nm}$ obtenue à partir de différents capteurs en fonction de la latitude le long de la sous-trace Calipso. Pour Airs et OMI, les informations complémentaires sont extraites de la climatologie fournie par le modèle Gocart. Un facteur 4 est appliqué sur les mesures de Airs pour les rapprocher des épaisseurs optiques à $500 \mathrm{~nm}$. D'après DeSouza-Machado et al. (2010). 


\section{Produire de nouvelles bases de données}

La prise en compte de processus de plus en plus complexes dans les modèles de chimie-transport entraîne le besoin d'établir des bases de données sur de nouvelles variables. Le calcul des émissions de feux de végétation et d'aérosols minéraux en est un bon exemple.

Les modèles régionaux de qualité de l'air ont besoin de connaître précisément la localisation d'un feu et son évolution dans le temps à une fréquence horaire. Les feux eux-mêmes étant peu prévisibles, une observation continue est indispensable. En ce sens, la disponibilité d'observations satellitaires est essentielle. Un service Copernicus spécifique est dédié à la production et à la mise à jour quotidienne d'une telle base de données d'émission de feux (GFED). Celle-ci sert à produire des données de forçage précises pour les modèles de prévision.

Les données satellitaires permettent tout d'abord de localiser les feux et d'estimer leur intensité. Elles permettent aussi de fournir des bases de données précises sur la nature de la végétation qui peut brûler et donc d'estimer la charge en combustible. On peut désormais estimer la contribution des feux de végétation dans le bilan de la pollution mesurée très en aval de feux importants. Un autre exemple de l'apport du satellite pour l'étude des feux de végétation consiste à corriger les flux émis a priori avec des observations par satellite du panache de pollution associé. Par exemple, à partir de Iasi (CO) et Modis (épaisseur optique en aérosols), il a été possible de revoir ces émissions au-dessus de la Russie européenne et de la Sibérie (R'honi et al., 2013 ; Konovalov et al., 2014). Les simulations réalisées avec le modèle Chimere ont permis de mieux restituer la qualité de l'air observée autour de Moscou pendant les grands feux de l'été 2010 (avec des concentrations de $\mathrm{PM}_{10}$ atteignant $800 \mu \mathrm{g} \mathrm{m}^{-3}$ et de $\mathrm{CO}$ atteignant $\left.10000 \mu \mathrm{g} \mathrm{m}^{-3}\right)$.

L'altitude d'injection des feux est un autre paramètre essentiel pour la modélisation de leur impact sur la qualité de l'air. L'énergie dégagée par le feu peut en effet induire ou renforcer un mouvement convectif, ce qui implique une injection des émissions à haute altitude pour les feux les plus intenses. Même si les observations satellitaires apportent une contrainte sur l'intensité des feux, ce phénomène reste difficile à modéliser. Des observations des structures verticales des panaches (avec MISR ou Caliop) permettent de contraindre directement l'altitude d'injection. La figure 5 montre la comparaison entre des données de $\mathrm{PM}_{2.5}$ mesurées à la surface proche de feux dans les Balkans pendant l'été 2012, ainsi que la simulation de cet événement avec le modèle Chimere avec des émissions de feux observées par satellite et une altitude d'injection dans la couche limite ou selon un profil observé par MISR. Une injection en altitude diminue mécaniquement la concentration en surface, améliorant l'accord avec les observations. Cette étude montre clairement que bien estimer l'altitude d'injection est critique pour les études de la qualité de l'air. Cependant, le manque de couverture spatiale des instruments permettant cette mesure de l'altitude des panaches ne permet pas pour le moment d'apporter systématiquement une contrainte robuste aux modèles.

Les émissions d'aérosols minéraux ont longtemps été calculées à partir de bases de données de sol ou de surface faiblement résolues et très approximatives. Le satellite permet de déduire des paramètres clés pour le calcul de ces émissions, comme la hauteur de rugosité de saltation et l'érodabilité, déduites des réflectances Parasol (Prigent et al., 2012). L'ajout dans les modèles de ce niveau de précision a permis d'accroître la variabilité des concentrations simulées par le modèle et de lui permettre d'être plus proche de ce qu'il est nécessaire de calculer lorsque l'on veut réaliser des prévisions sur plusieurs jours et à échéance horaire.
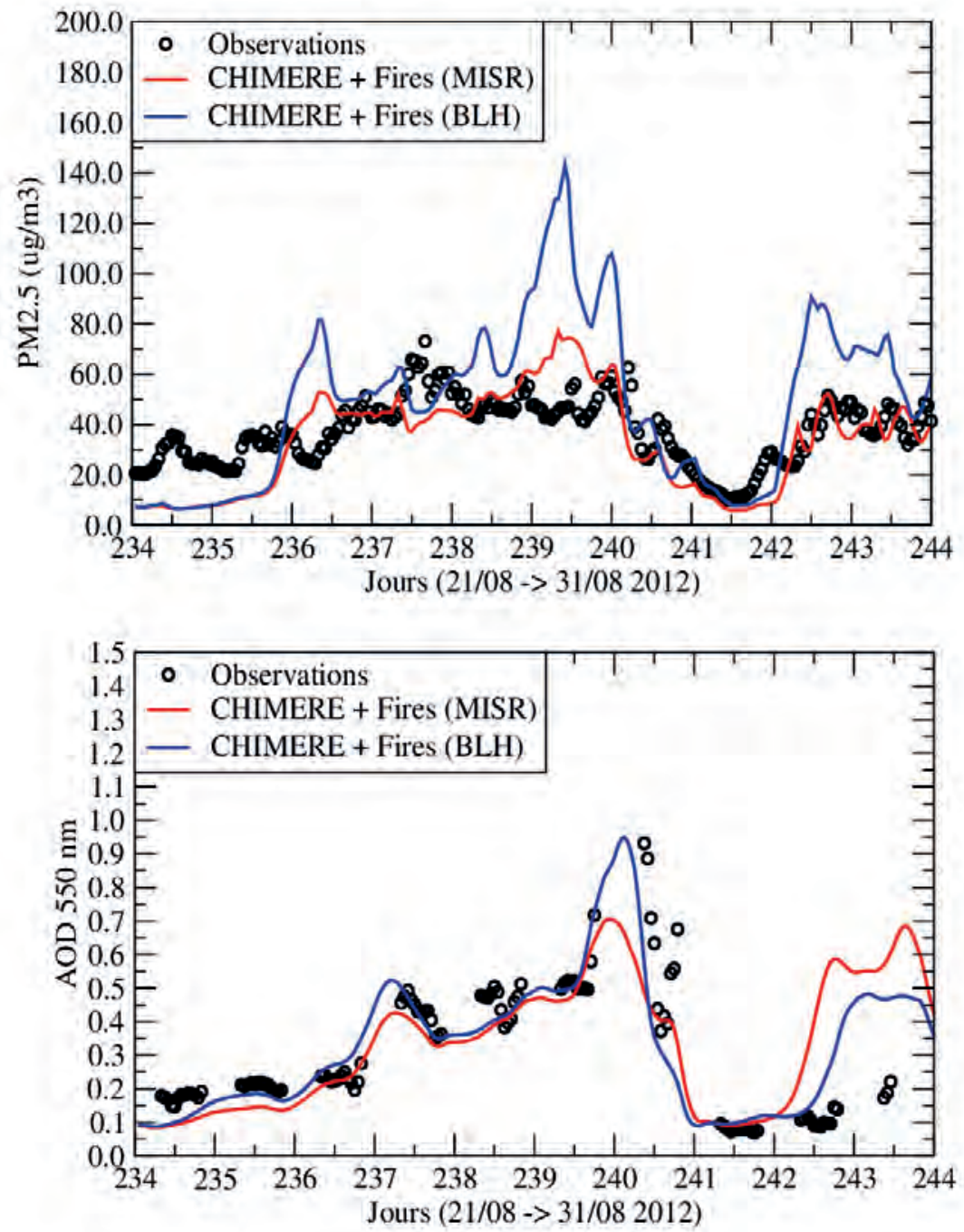

Figure 5. Comparaison entre les simulations réalisées par le modèle Chimere avec une hauteur d'injection des feux déduite de MISR ou restreinte à la couche limite (BLH) avec des observations pour (en haut) la concentration de $\mathrm{PM}_{2.5}$ de surface à la station de Skopje (Macédoine) proche des feux et (en bas) l'épaisseur optique en aérosols à $550 \mathrm{~nm}$ mesurée et observée à la station de Timisoara (Roumanie) dans le panache transporté. D'après la thèse de Géraldine Réa (2015). 


\section{La modélisation des observables satellitaires}

La comparaison entre simulation et observation nécessite dans un premier temps de s'assurer que des quantités comparables sont analysées. La première étape est de considérer des données colocalisées et simultanées. Dans le cas de l'analyse d'observations satellitaires, il est souvent nécessaire d'aller plus loin, et on devra estimer ce que l'instrument aurait observé pour la situation modélisée, c'est-à-dire l'observable correspondant aux concentrations simulées. Une comparaison quantitative pourra ensuite être menée. Cette approche est appelée OSSE pour Observing System Simulation Experiments et recouvre un large éventail de techniques de modélisation (Timmermans et al., 2015).

En effet, le principe de la télédétection implique que les mesures réalisées correspondent à une information intégrée sur l'état de l'atmosphère à un instant donné. L'observation de la troposphère étant réalisée au nadir (de l'espace vers le sol), le rayonnement mesuré depuis l'espace représente toute la colonne atmosphérique au-dessous de l'instrument. Dans le cas d'une télédétection passive, on analysera le plus souvent une information intégrée selon l'axe vertical du contenu en gaz trace (colonne troposphérique) ou en aérosols (via leur signature optique caractérisée par leur épaisseur optique, par exemple). La simulation d'observable consistera donc à calculer l'équivalent de la colonne atmosphérique intégrée à partir de profils verticaux de concentrations simulées.

Pour certains instruments cependant, le contenu en information n'est pas uniforme sur la verticale. On a par exemple un pic de sensibilité dans la troposphère libre pour la mesure dans l'infrarouge thermique (type Iasi) et une sensibilité à la couche limite qui dépend fortement du contraste thermique (Clerbaux et al., 2009). Si l'instrument manque de sensibilité dans la couche limite, une comparaison directe avec une simulation pour une zone urbaine dans laquelle la majeure partie de la pollution est localisée n'a pas de sens. La variation de la sensibilité sur la verticale ainsi que la corrélation entre les niveaux d'inversion (l'inversion à un niveau donné n'est pas indépendante des concentrations réelles aux niveaux voisins) sont souvent caractérisées grâce aux fonctions de lissage, fournies aux utilisateurs au même titre que les concentrations atmosphériques et les erreurs associées. L'observable calculée par le modèle sera alors obtenue en combinant les profils du modèle par les fonctions de lissage. L'observable sera alors soit une colonne intégrée, soit un profil sur les niveaux d'inversion tenant compte de la fonction de lissage, en fonction de la résolution spectrale et donc verticale de l'observation satellitaire.

Un exemple récent est l'utilisation de produits multispectraux dont la synergie des bandes dans l'infrarouge (Iasi) et l'ultraviolet (Gome-2) permet l'observation de panaches d'ozone situés au-dessous de $3 \mathrm{~km}$ d'altitude, avec une sensibilité maximale proche de $2 \mathrm{~km}$ (figure 6a) (Cuesta et al., 2013). Cela permet une comparaison directe et quantitative avec la simulation Chimere correspondante (figure 6b). Si la comparaison montre un bon accord entre observations et modèle, les différences sont attribuables à l'erreur du modèle d'une part et aux variations de la sensibilité de la combinaison de Iasi et Gome-2 sur la verticale d'autre part. Afin de se rapprocher au mieux des observations, les simulations Chimere sont alors optimisées en utilisant les fonctions de lissage du produit satellitaires. Concernant les aérosols, des méthodes de simulateurs d'observables permettent de calculer à partir des concentrations d'aérosols simulées ce qui serait observé par un lidar comme Caliop (Stromatas et al., 2012).

\section{Assimiler \\ les observations satellitaires}

À l'instar de ce qui se fait en météorologie, les observations satellitaires peuvent être utilisées pour améliorer directement les résultats des simulations de chimie-transport. Dans le cas de la composition de l'atmosphère, cette utilisation conjointe des modèles et des observations satellitaires peut permettre d'obtenir des champs analysés (c'est à dire des champs de concentration hybridant les résultats du modèle avec les mesures
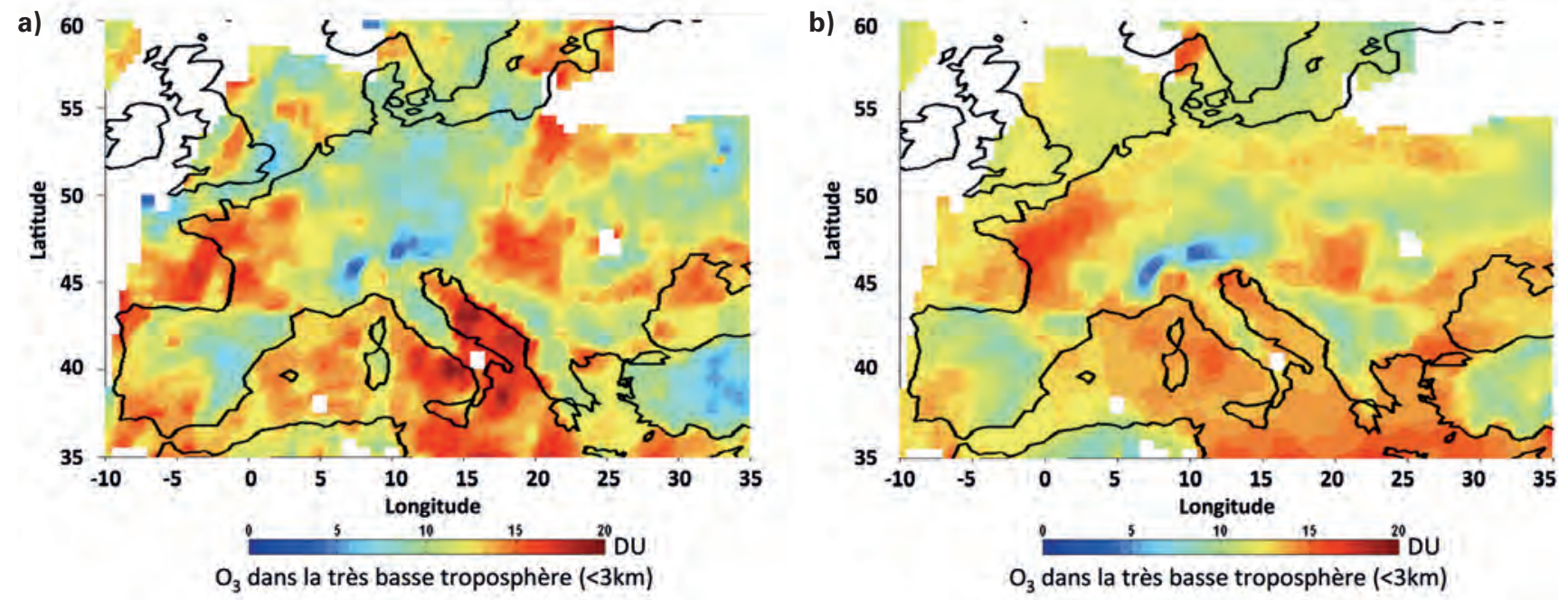

Figure 6. Distribution de la colonne d'ozone dans la très basse troposphère (entre la surface et $3 \mathrm{~km}$ d'altitude) en unités Dobson (DU) durant un événement de pollution le 19 août 2009 en Europe à partir : (a) du produit multispectral lasi + Gome-2 par synergie des spectres lasi dans l'infrarouge et Gome-2 dans I'ultraviolet ; (b) des simulations du modèle de chimie-transport Chimere avec lissage vertical par les fonctions de lissage du produit lasi + Gome-2. 
disponibles), mais aussi des inventaires d'émissions corrigés via la modélisation inverse.

\section{La modélisation inverse des émissions}

Une part significative de l'incertitude des modèles provient des émissions utilisées en forçage. Les inventaires d'émissions sont traditionnellement construits en agrégeant de nombreuses données. Un moyen d'affiner ces inventaires consiste à utiliser des techniques de modélisation inverse : on estime les émissions qu'il aurait fallu pour simuler au mieux les concentrations mesurées. Fondées sur des approches bayésiennes, ces méthodes ont été initialement développées pour des modélisations globales et des espèces peu réactives $\left(\mathrm{CO}_{2}, \mathrm{CH}_{4}, \mathrm{CO}\right)$ pour lesquelles les concentrations sont linéairement liées aux émissions.

À l'échelle régionale, cette approche est plus difficile à mettre en œuvre : pour les espèces chimiques impliquées dans la pollution atmosphérique, les sources sont plus difficiles à temporaliser, la chimie est plus rapide et non linéaire, la météorologie est plus variable. Le risque est alors de reporter l'ensemble des erreurs de modélisation dans des flux émis corrigés. Les travaux actuels utilisant des mesures satellitaires sont surtout fondés sur des inversions de $\mathrm{NO}_{\mathrm{X}}$ : on peut considérer en première approximation que cette espèce est surtout présente dans la basse troposphère et donc que la mesure satellitaire intégrée verticalement (par Gome, Sciamachy, OMI ou Gome-2) est assez proche de la concentration de surface. Plus récemment, d'autres espèces chimiques ont été inversées, notamment l'isoprène pour les émissions biogéniques. Pour les émissions anthropiques, les observations d'ammoniac de Iasi ont permis de revoir les émissions agricoles en Europe comme le montre la figure 7 (Fortems-Cheiney et al., 2016). Les recherches actuelles pour la modélisation inverse régionale sont amenées à se développer en s'appuyant sur les futurs instruments satellitaires, Tropomi et Iasi-NG, qui devraient permettre d'aller vers des résolutions spatiales plus fines et de contraindre de nouvelles espèces gazeuses.

\section{L'assimilation pour ré-analyser des champs de concentrations simulés}

Si l'utilisation des observations de surface pour l'assimilation dans les modèles de qualité de l'air s'est fortement développée au cours de la dernière décennie (Marécal et al., 2015), le cas des observations satellitaires reste plus complexe : le problème à traiter implique une grande variabilité d'échelles spatiales et temporelles (en fonction du capteur et de la durée de vie de l'espèce), la résolution verticale reste cruciale lorsque l'on cherche à assimiler une espèce chimique dont le profil vertical est très variable et donc lorsque les valeurs dans la basse troposphère ne peuvent pas être représentées de manière réaliste par des colonnes intégrées. De plus, l'incertitude sur les quantités inversées (notamment pour retrouver des concentrations à partir de luminances) reste aujourd'hui une limitation forte à une assimilation robuste.

Cependant, deux espèces chimiques gazeuses font l'objet de nombreuses études d'assimilation avec un gain net sur les résultats : l'ozone et le $\mathrm{CO}$.

Pour l'ozone, l'accent est mis sur l'assimilation de colonnes troposphériques (comme celle issues de Sciamachy ou OMI) et/ou des profils disponibles pour la basse stratosphère voire la haute troposphère (Mipas ou MLS, plus récemment). Les analyses produites montrent généralement une amélioration des concentrations d'ozone estimées dans la haute troposphère/basse stratosphère par rapport aux champs non contraints par les observations. Par contre, dans la troposphère, les améliorations sont faibles lorsque l'on n'observe pas une augmentation des biais entre modèle et observations (Massart et al., 2009). À l'heure actuelle, dans la troposphère libre, les travaux d'assimilation les plus encourageants sont fondés sur l'utilisation des produits infrarouge issus des instruments TES et Iasi qui permettent d'avoir une quantité d'information plus importante dans la a)

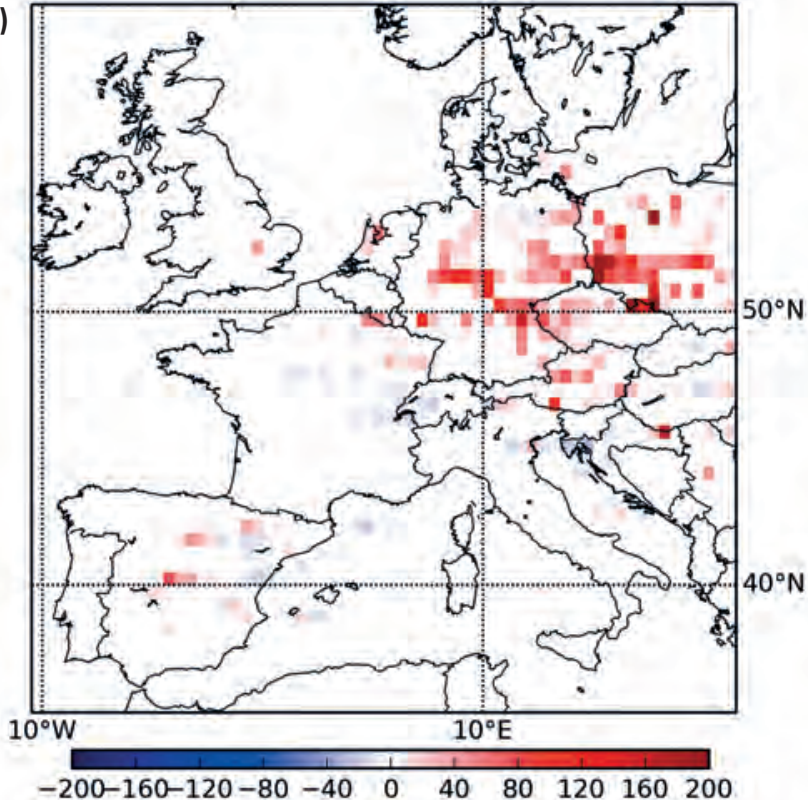

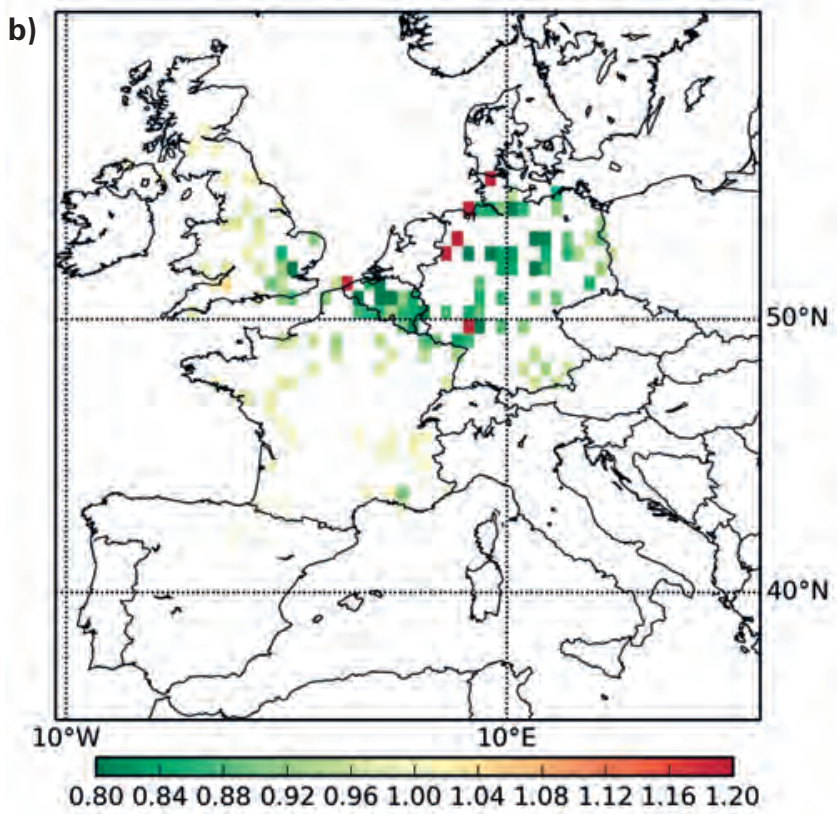

Figure 7. (a) Différence (\%) entre les émissions d'ammoniac inversées par lasi (NH3-SAT) et les émissions a priori Emep. (b) Le rapport entre les biais calculés entre les concentrations de $\mathrm{PM}_{2.5}$ simulées avec Chimere et les mesures de surface AirBase, avec ou sans émissions de NH3-SAT, montre une amélioration pour quasiment l'ensemble des stations AirBase quand les émissions NH3-SAT sont utilisées. 
troposphère qu'avec d'autres capteurs. L'assimilation a pu se faire sur des colonnes partielles, se focalisant sur la basse troposphère (Coman et al., 2012). Cependant, la faible sensibilité de ces observations dans la couche limite et leur faible résolution verticale restent des freins à leur utilisation plus systématique pour les systèmes opérationnels de qualité de l'air. Le cas spécifique de l'ozone, polluant avec des concentrations plus faibles dans la troposphère que dans la stratosphère et avec les données satellitaires, par définition, peu informatives près de la surface, font que ces études n'ont pas encore permis d'obtenir des assimilations utilisables pour des analyses fines de cas de pollution atmosphérique.

Pour le CO, des assimilations de données ont été réalisées en utilisant à la fois Iasi et Mopitt. Ce dernier, avec ses canaux sensibles à la fois dans l'infrarouge thermique et dans le proche infrarouge, a une sensibilité améliorée vers la surface et près des sources de $\mathrm{CO}$, mais il a une moins bonne couverture spatiale que Iasi qui apporte une information complémentaire à l'échelle globale (transport) et a une meilleure sensibilité dans la troposphère moyenne. Les mesures des deux instruments sont assimilées (Inness et al., 2015) en temps réel et fournissent aujourd'hui les prévisions opérationnelles du service Copernicus (Wagner et al., 2015). Ces assimilations sont basées sur des plates-formes LEO (Low Earth Orbit) et il n'existe pas encore actuellement de plate-forme géostationnaire pour ces espèces chimiques. Des études récentes, fondées sur des expériences de simulation de système d'observation (Hache et al., 2014), ont montré l'apport positif qu'aurait ce type d'instrument pour la surveillance de la pollution et la qualité de l'air à l'échelle régionale ; des projets de ce type sont aujourd'hui en préparation.

Des études d'assimilation sont aussi réalisées pour les aérosols. Comme pour les gaz, ces études sont plus souvent à l'échelle globale que régionale et offrent donc des informations de qualité de l'air à une résolution encore insuffisante. La plupart des efforts sont axés sur l'assimilation d'épaisseur optique d'aérosol (par exemple, Benedetti et al., 2009) : il reste encore à développer des systèmes qui assimileront directement des concentrations d'espèces chimiques différenciées, l'AOD représentant partiellement les effets des aérosols sur l'état de l'atmosphère.

\section{L'assimilation pour améliorer les prévisions à court terme}

L'enjeu de l'assimilation des polluants dans les modèles de prévision à court terme (un à trois jours) est de corriger l'état initial de la prévision. Ceci afin de permettre de combler la dérive de la prévision qui résulte des incertitudes liées à la représentation des processus dans les modèles et également aux données d'entrée (météorologie et émissions principalement).

Un cas récent montre l'intérêt de l'assimilation dans les modèles de prévision (Engelen et al., 2015). Un épisode de pollution sur l'Europe de l'Ouest caractérisé par des concentrations élevées en particules est survenu au mois de septembre 2014. Il a été initié par des émissions naturelles de $\mathrm{SO}_{2}$ du volcan islandais Bárðarbunga, non intégrées dans les modèles de prévision régionale car exceptionnelles. L'assimilation de données $\mathrm{OMI}$ de $\mathrm{SO}_{2}$ dans les prévisions globales de composition chimique de l'atmosphère a permis d'intégrer cette contribution dans les prévisions régionales via leurs conditions aux limites et d'estimer son effet sur la formation des particules en Europe et en France, améliorant ainsi la qualité de la prévision sur quelques jours.

Le défi futur sera d'initialiser les prévisions de la qualité de l'air via l'assimilation de données dans tous types de modèles (du global jusqu'au local en passant par le régional) et que cette information transmise perdure dans la prévision et améliore les performances de celle-ci assez systématiquement, ce qui pour le moment n'est pas réalisé de façon satisfaisante. Cela passera certainement par l'assimilation conjointe de données satellitaires et in situ. Des informations multicapteurs devront permettre de mieux décrire les polluants atmosphériques dans la couche limite et transmettre au modèle une information spatiale concernant la composition chimique de l'aérosol.

\section{Le service Copernicus atmosphère}

Les services développés dans le cadre du programme Copernicus, initialement nommé GMES (Global Monitoring for Environment and Security), sont des systèmes opérationnels d'évaluation, de prévision et d'analyse des pollutions et des risques, afin de faciliter l'aide à la décision des autorités européennes, nationales voire locales, et d'appuyer la conception de législations environnementales. Ces services couvrent les différents compartiments de l'environnement, dont l'atmosphère. Le service atmosphère, récemment baptisé Cams (pour Copernicus Atmosphere Monitoring Service) par le Centre européen pour les prévisions météorologiques à moyen terme qui en assure la coordination par délégation pour la Commission européenne, couvre aussi bien les questions de composition chimique de l'atmosphère à l'échelle globale (gaz à effet de serre, gaz réactifs, aérosols) que de qualité de l'air à l'échelle régionale (Europe) et d'exposition aux rayonnements ultraviolets. Le développement de ces services s'est fait avec le soutien des mesures satellitaires existantes au cours des programmes de recherche européens Gems et Macc.

Aujourd'hui le service atmosphère est pleinement opérationnel et dispose de plusieurs composantes qui ont une utilisation quotidienne des mesures satellites pour fournir leurs informations. C'est notamment crucial pour l'élaboration des émissions issues des feux de biomasse, pour la prévision des gaz réactifs, pour la prévision des aérosols et pour l'élaboration d'information sur la qualité de l'air.

\section{Les perspectives d'utilisation des observations satellitaires}

Les perspectives d'utilisation future des observations satellitaires se placent à deux niveaux : (1) l'opérationnalisation des utilisations « recherche » décrites tout le long de cet article, notamment au sein du système européen Cams et (2) la recherche sur des nouvelles applications à partir de missions existantes ou nouvelles.

L'initiative Cams a permis d'établir les bases d'un système pré-opérationnel qui exploite les données satellitaires pour la prévision de la pollution atmosphérique aux échelles régionales et globales, tant au niveau de l'organisation de l'interface observationmodèle que pour l'incitation à mettre 
au point des filières d'instruments qui fournissent des informations en temps réel et sur le long terme. Dans le futur, les observations issues des sondeurs atmosphériques seront utilisées de façon opérationnelle pour : améliorer les inventaires d'émissions, produire des analyses de la composition chimique de l'atmosphère et améliorer la prévision à court terme de la qualité de l'air. L'amélioration des inventaires d'émissions (par exemple $\mathrm{NO}_{2}, \mathrm{NH}_{3}$, $\mathrm{CO}$, certains composés organiques volatils) pourra se faire à un rythme plus fréquent, avec une réactivité que ne permettent pas les approches fondées sur des comptages source par source (délai d'environ 3 ans) et donnera accès aux tendances pluriannuelles des émissions. Cela permettra de suivre l'efficacité de la mise en place des mesures de réduction des émissions de polluants. Une des difficultés sera d'avoir accès aux données en temps réel pour pouvoir les assimiler en routine. Du côté de la modélisation, les limitations sont liées au temps de calcul nécessaire pour assimiler des grands jeux d'observation et au fait que l'information fournie par les données satellitaires est intégrée sur la verticale, avec une information limitée ou absente au niveau de la couche limite atmosphérique et de la surface, là où l'essentiel de la pollution se développe et concerne les populations.

Les instruments du futur sont en cours de construction, en particulier les Sentinelles qui sont la composante satellitaire de Copernicus. Le tableau 2 résume les missions décidées qui fourniront l'essentiel des observations de sondage pour les gaz et les particules liées à la qualité de l'air. Elles fourniront des informations nouvelles, car les sondeurs seront plus performants (avec une meilleure précision, davantage de sensibilité verticale, en particulier vers la surface) et plus fréquentes. Par ailleurs, d'ici quelques années, les plateformes météorologiques qui surveillent la Terre depuis 1'orbite géostationnaire accueilleront des sondeurs dédiés à la chimie atmosphérique, fournissant des mesures avec une fréquence de 15 minutes à une heure, au lieu de deux passages par jour au mieux aujourd'hui. Cette meilleure couverture temporelle permettra de pleinement résoudre la variabilité diurne de la pollution, de dériver les flux de polluants (entre les continents, entre les pays en combinaison avec les données de vent) et enfin de dériver de nouveaux produits porteurs d'information à partir de rapports entre différentes mesures colocalisées.
Tableau 2. Principales caractéristiques des futures missions satellitaires pertinentes pour l'étude de la qualité de l'air.

$\begin{array}{llll}\text { Nom } & \text { Spécifications } & \text { Polluants } & \text { Nouvelles performances } \\ \text { du sondeur } & \text { instrumentales } & \text { mesurés } & \text { attendues }\end{array}$

\section{Orbite polaire}

\begin{tabular}{|c|c|c|c|}
\hline $\begin{array}{l}\text { Tropomi } \\
\text { Sentinel-5 } \\
\text { précurseur }\end{array}$ & $\begin{array}{l}\text { Spectromètre UV-vis- } \\
\text { NIR } \\
\text { Pixel : } 10 \text { km x } 10 \text { km } \\
\text { Fauchée : } 2300 \text { km }\end{array}$ & $\begin{array}{l}\mathrm{NO}_{2}, \mathrm{O}_{3}, \mathrm{CO}, \mathrm{SO}_{2} \\
\text { (PM ?) }\end{array}$ & $\begin{array}{l}\text { Héritage } 0 M \mathrm{Ml} \text { avec la taille } \\
\text { du pixel et précision améliorées }\end{array}$ \\
\hline $\begin{array}{l}\text { UVNS } \\
\text { Sentinel-5 } \\
\text { /Metop-SG }\end{array}$ & $\begin{array}{l}\text { Spectromètre UV-vis } \\
\text { Pixel : 7,5 km x 7,5 km } \\
\text { Fauchée : } 2670 \mathrm{~km}\end{array}$ & $\mathrm{NO}_{2}, \mathrm{O}_{3}, \mathrm{SO}_{2}, \mathrm{CH}_{2} \mathrm{O}$ & $\begin{array}{l}\text { Héritage Gome-2 avec taille } \\
\text { du pixel améliorée }\end{array}$ \\
\hline $\begin{array}{l}\text { lasi-NG } \\
\text { Sentinel-5 } \\
\text { /Metop-SG }\end{array}$ & $\begin{array}{l}\text { Spectromètre } \\
\text { à transformée } \\
\text { de Fourier } \\
\text { TIR }(15,5-3,62 \mu \mathrm{m}) \\
\text { Pixel : } 12 \mathrm{~km} \\
\text { de diamètre } \\
\text { Fauchée : } 2200 \text { km }\end{array}$ & $\begin{array}{l}\mathrm{CO}, \mathrm{O}_{3}, \mathrm{NH}_{3} \\
\mathrm{SO}_{2}, \mathrm{CH}_{3} \mathrm{OH} \\
\mathrm{HCOOH} \\
\text { (PAN) }\end{array}$ & $\begin{array}{l}\text { Héritage lasi avec la sensibilité } \\
\text { vers la surface et la précision } \\
\text { améliorée }\end{array}$ \\
\hline $\begin{array}{l}3 \mathrm{Ml} \\
\text { Sentinel-5 } \\
\text { /Metop-SG }\end{array}$ & $\begin{array}{l}\text { Polarimètre } \\
\text { multispectral, } \\
\text { multidirectionnel, } \\
\text { multipolarisation } \\
\text { (UV, vis, NIR) } \\
\text { 10-14 angles } \\
\text { d'observation } \\
\text { Pixel : } 4 \mathrm{~km} \times 4 \mathrm{~km} \\
\text { Fauchée : } 2400 \mathrm{~km}\end{array}$ & $\begin{array}{l}\text { Aérosols : } \\
\text { distribution } \\
\text { en taille (fin/grossier, } \\
\text { sphérique/ } \\
\text { non sphérique), } \\
\text { AOD, indice de } \\
\text { réfraction, albédo } \\
\text { de simple diffusion }\end{array}$ & $\begin{array}{l}\text { Héritage Polder avec la } \\
\text { spéciation des aérosols, } \\
\text { l'altitude et la précision } \\
\text { améliorée }\end{array}$ \\
\hline
\end{tabular}

\section{Orbite géostationnaire}

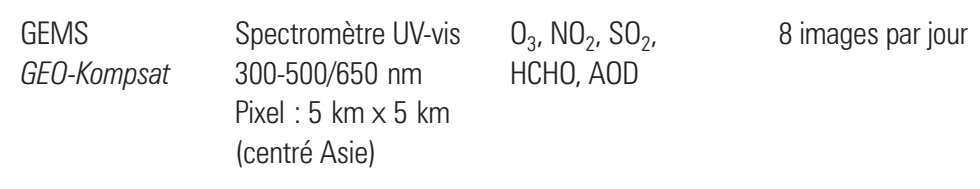

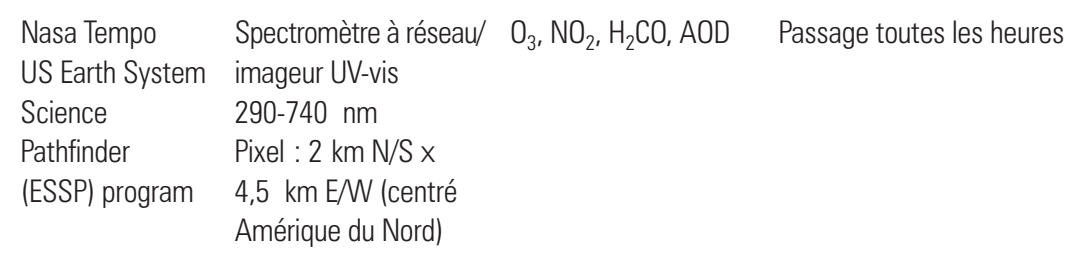

\begin{tabular}{|c|c|c|c|}
\hline $\begin{array}{l}\text { EU UVN } \\
\text { Sentinel-4 } \\
\text { /Meteosat } \\
\text { Third Generation }\end{array}$ & $\begin{array}{l}\text { Spectromètre UV-vis- } \\
\text { NIR } \\
\text { UV-vis } 305-500 \mathrm{~nm} \\
\text { NIR } 750-775 \mathrm{~nm} \\
\text { Pixel } \leq 8 \mathrm{~km} \times 8 \mathrm{~km} \\
\text { (centré Europe-Afrique) }\end{array}$ & $\mathrm{O}_{3}, \mathrm{NO}_{2}, \mathrm{SO}_{2}$ & $\begin{array}{l}\text { Passage toutes les } \\
\text { 30-60 minutes } \\
\text { au-dessus de l'Europe }\end{array}$ \\
\hline $\begin{array}{l}\text { EU IRS } \\
\text { Sentinel-4 } \\
\text { /Meteosat } \\
\text { Third } \\
\text { Generation }\end{array}$ & $\begin{array}{l}\text { Spectromètre } \\
\text { à transformée } \\
\text { de Fourier } \\
\text { TIR 4,44 à } 14,7 \mu \mathrm{m} \\
\text { Pixel : } 4 \mathrm{~km} \times 4 \mathrm{~km} \\
\text { (centré Europe-Afrique) }\end{array}$ & $\mathrm{CO}, \mathrm{O}_{3}, \mathrm{NH}_{3}$ & $\begin{array}{l}\text { Passage toutes les } \\
15-30 \text { minutes } \\
\text { au-dessus de l'Europe }\end{array}$ \\
\hline
\end{tabular}




\section{Bibliographie}

Benedetti A., Morcrette J.-J., Boucher O., Dethof A., Engelen R., Fisher M., Flentje H., Huneeus N., Jones L., Kaiser J., S.Kinne, A.Mangold, M.Razinger, A.J.Simmons and M.Suttie, 2009. Aerosol analysis and forecast in the European Centre for Medium-range Weather forecasts integrated forecast system: 2. Data assimilation. J. Geophys. Res. Atm., 114, D13205. doi:10.1029/2008JD011115

Bessagnet B., Menut L., Aymoz G., Chepfer H., Vautard R., 2008. Modelling dust emissions and transport within Europe: the Ukraine March 2007 event. J. Geophys. Res, 113, D15202. doi: 10.1029/2007JD009541

Boichu M., Clarisse L., Péré J.-C., Herbin H., Goloub P., Thieuleux F., Ducos F., Clerbaux C., Tanré D., 2015. Temporal variations of flux and altitude of sulfur dioxide emissions during volcanic eruptions: implications for long-range dispersal of volcanic clouds. Atmos. Chem. Phys., 15, 8381-8400. doi: 10.5194/acp-15-8381-2015

Chazette P., Bocquet M., Royer P., Winiarek V., Raut J.-C., Labazuy P., Gouhier M., Lardier M., Cariou J.-P., 2012. Eyjafjallajökull ash concentrations derived from both lidar and modeling. J. Geophys. Res., 117, D00U14. doi: 10.1029/2011JD015755

Clerbaux C., Boynard A., Clarisse L., George M., Hadji-Lazaro J., Herbin H., Hurtmans D., Pommier M., Razavi A., Turquety S., Wespes C., Coheur P.-F., 2009. Monitoring of atmospheric composition using the thermal infrared IASI/MetOp sounder. Atmos. Chem. Phys., 9, 6041-6054.

Coman A., Foret G., Beekmann M., Eremenko M., Dufour G., Gaubert B., Ung A., Schmechtig C., Flaud J.-M., Bergametti G., 2012. Assimilation of IASI partial tropospheric columns with an Ensemble Kalman Filter over Europe. Atmos. Chem. Phys., 12, 2513-2532. doi: 10.5194/acp-12-2513-2012

Cuesta J., Eremenko M., Liu X., Dufour G., Cai Z., Höpfner M., von Clarmann T., Sellitto P., Foret G., Gaubert B., Beekmann M., Orphal J., Chance K., Spurr R., Flaud J.-M., 2013. Satellite observation of lowermost tropospheric ozone by multispectral synergism of IASI thermal infrared and GOME-2 ultraviolet measurements over Europe. Atmos. Chem. Phys., $13,9675-9693$.

Cuesta J., Eremenko M., Flamant C., Dufour G., Laurent B., Bergametti G., Höpfner M., Orphal J., Zhou D., 2015. Three-dimensional distribution of a major desert dust outbreak over East Asia in March 2008 derived from IASI satellite observations. J. Geophys. Res. Atmos., 120, 7099-7127. doi: 10.1002/2014JD022406

Deeter M.N., Edwards D.P., Gille J.C., Worden H.M., 2015. Information content of MOPITT CO profile retrievals: Temporal and geographical variability. J. Geophys. Res. Atmos., 120, 12723-12738. doi: 10.1002/2015JD024024

Doche C., Dufour G., Foret G., Eremenko M., Cuesta J., Beekmann M., Kalabokas P., 2014. Summertime tropospheric-ozone variability over the Mediterranean basin observed with IASI. Atmos. Chem. Phys., 14, 10589-10600. doi: 10.5194/acp-14-10589-2014

Engelen R., Flemming J., Hedelt P., Inness A., Suttie M., Valks P., 2015. Assimilating volcanic $\mathrm{SO}_{2}$ satellite data in the Copernicus atmosphere monitoring service global data assimilation system. In: ATMOS 2015 Abstract Book. ESA ATMOS 2015, 8-12 June 2015, Crète, Grèce.

Fortems-Cheiney A., Dufour G., Hamaoui-Laguel L., Foret G., Siour G., Van Damme M., Meleux F., Coheur P.-F., Clerbaux C., Clarisse L., Favez 0., Wallasch M., Beekmann M., 2016. Unaccounted variability in $\mathrm{NH}_{3}$ agricultural sources detected by IASI contributing to European spring haze episode. Geophys. Res. Lett., 43, 5475-5482. doi: 10.1002/2016GL069361

George M., Clerbaux C., Bouarar I., Coheur P.-F., Deeter M. N., Edwards D.P., Francis G., Gille J.C., Hadji-Lazaro J., Hurtmans D., Inness A., Mao D., Worden H.M., 2015. An examination of the long-term CO records from MOPITT and IASI: comparison of retrieval methodology. Atmos. Meas. Tech., 8, 4313-4328. doi: 10.5194/amt-8-4313-2015

Guenther A.B., Jiang X., Heald C.L., Sakulyanontvittaya T., Duhl T., Emmons L.K., Wang X., 2012. The model of emissions of gases and aerosols from nature version 2.1 (MEGAN2.1): an extended and updated framework for modeling biogenic emissions. Geosci. Model Dev., 5, 1471-1492. doi: 10.5194/gmd-5-1471-2012

Hache E., Attié J.-L., Tourneur C., Ricaud P., Coret L., Lahoz W.A., El Amraoui L., Josse B., Hamer P., Warner J., Liu X., Chance K., Höpfner M., Spurr R., Natraj V., Kulawik S., Eldering A., Orphal J., 2014. The added value of a visible channel to a geostationary thermal infrared instrument to monitor ozone for air quality. Atmos. Meas. Tech., 7, 2185-2201. doi: 10.5194/amt-7-2185-2014

Inness A., Blechschmidt A.-M., Bouarar I., Chabrillat S., Crepulja M., Engelen R.J., Eskes H., Flemming J., Gaudel A., Hendrick F., Huijnen V., Jones L., Kapsomenakis J., Katragkou E., Keppens A., Langerock B., de Mazière M., Melas D., Parrington M., Peuch V.H., Razinger M., Richter A., Schultz M.G., Suttie M., Thouret V., Vrekoussis M., Wagner A., Zerefos C., 2015. Data assimilation of satellite-retrieved ozone, carbon monoxide and nitrogen dioxide with ECMWF's Composition-IFS. Atmos. Chem. Phys., 15, 5275-5303. doi: 10.5194/acp-15-5275-2015

Konovalov I.B., Berezin E.V., Ciais P., Broquet G., Beekmann M., Hadji-Lazaro J., Clerbaux C., Andreae M.O., Kaiser J.W., Schulze E.-D., 2014. Constraining CO 2 emissions from open biomass burning by satellite observations of co-emitted species: a method and its application to wildfires in Siberia. Atmos. Chem. Phys., 14, 10383-10410. doi: 10.5194/acp-14-10383-2014

Marécal M., Peuch V.-H., Andersson C., Andersson S., Arteta J., Beekmann M., Benedictow A., Bergström R., Bessagnet B., Cansado A., Chéroux F., Colette A., Coman A., Curier R.L., Denier van der Gon H.A.C., Drouin A., Elbern H., Emili E., Engelen R.J., Eskes H.J., Foret G., Friese E., Gauss M., Giannaros C., Guth J., Joly M., Jaumouillé E., Josse B., Kadygrov N., Kaiser J.W., Krajsek K., Kuenen J., Kumar U., Liora N., Lopez E., Malherbe L., Martinez I., Melas D., Meleux F., Menut L., Moinat P., Morales T., Parmentier J., Piacentini A., Plu M., Poupkou A., Queguiner S., Robertson L., Rouil L., Schaap M., Segers A., Sofiev M., Tarasson L., Thomas M., Timmermans R., Valdebenito A., van Velthoven P., van Versendaal R., Vira J., Ung A., 2015. A regional air quality forecasting system over Europe: the MACC-II daily ensemble production. Geosci. Model Dev., 8, 2777-2813. doi: 10.5194/gmd-8-2777-2015

Massart S., Clerbaux C., Cariolle D., Piacentini A., Turquety S., Hadji-Lazaro J., 2009. First steps towards the assimilation of IASI ozone data into the MOCAGE-PALM system. Atmos. Chem. Phys., 9, 5073-5091. doi: 10.5194/acp-9-5073-2009

Prigent C., Jimenez C., Catherinot J., 2012. Comparison of satellite microwave backscattering (ASCAT) and visible/near-infrared reflectances (PARASOL) for the estimation of aeolian aerodynamic roughness length in arid and semi-arid regions. Atmos. Meas. Tech., 5, 2703-2712. doi: 10.5194/amt-5-2703-2012

Rea G, S.Turquety, L.Menut, R.Briant, S.Mailler and G.Siour, 2015, Source contributions to summertime aerosols in the Euro-Mediterranean region. Atmos. Chem. Phys., 15, 8013-8036, doi:10.5194/acp-15-8013-2015

R'honi Y., Clarisse L., Clerbaux C., Hurtmans D., Duflot V., Turquety S., Ngadi Y., Coheur P.-F., 2013. Exceptional emissions of $\mathrm{NH}_{3}$ and HCOOH in the 2010 Russian wildfires. Atmos. Chem. Phys., 13, 4171-4181. doi: 10.5194/acp-13-4171-2013

Stromatas S., Turquety S., Menut L., Chepfer H., Cesana G., Péré J.-C., Bessagnet B., 2012. Lidar signal simulation for the evaluation of aerosols in chemistry-transport models. Geosci. Model Dev., 5, 1543-1564.

Safieddine S., Boynard A., Coheur P.-F., Hurtmans D., Pfister G., Quennehen B., Thomas J.L., Raut J.-C., Law K.S., Klimont Z., Hadji-Lazaro J., George M., Clerbaux C., 2014. Summertime tropospheric ozone assessment over the Mediterranean region using the thermal infrared IASI/MetOp sounder and the WRF-Chem model. Atmos. Chem. Phys., 14, 10119-10131. doi: 10.5194/acp-14-10119-2014.

Timmermans R.M.A., Lahoz W.A., Attié J.-L., Peuch V.-H., Curier R.L., Edwards D.P., Eskes H.J., Builtjes P.J.H., 2015. Observing System Simulation Experiments for air quality. Atmos. Env., 115, 199-213. doi: 10.1016/j.atmosenv.2015.05.032

Wagner A., Blechschmidt A.-M., Bouarar I., Brunke E.-G., Clerbaux C., Cupeiro M., Cristofanelli P., Eskes H., Flemming J., Flentje H., George M., Gilge S., Hilboll A., Inness A., Kapsomenakis J., Richter A., Ries L., Spangl W., Stein 0., Weller R., Zerefos C., 2015. Evaluation of the MACC operational forecast system - potential and challenges of global near-real-time modelling with respect to reactive gases in the troposphere. Atmos. Chem. Phys., 15, 14005-14030. doi: 10.5194/acp-15-14005-2015

Waquet F., Cornet C., Deuzé J.-L., Dubovik O., Ducos F., Goloub P., Herman M., Lapionak T., Labonnote L., Riedi J., Tanré D., Thieuleux F., Vanbauce C., 2013. Retrieval of aerosol microphysical and optical properties above liquid clouds from POLDER/PARASOL polarization measurements. Atmos. Meas. Tech., 6, 991-1016. 